\title{
Correlation of Serum-Soluble Triggering Receptor Expressed on Myeloid Cells-1 with Clinical Disease Activity in Inflammatory Bowel Disease
}

Jae Jun Park · Jae Hee Cheon · Bo Young Kim •

Duk Hwan Kim · Eun Soo Kim · Tae Il Kim •

Kyoung Ryul Lee $\cdot$ Won Ho Kim

Published online: 1 January 2009

(C) Springer Science+Business Media, LLC 2008

\section{Erratum to: Dig Dis Sci}

DOI 10.1007/s10620-008-0514-5

The following errors were published in this article.

In the upper left corner of Fig. 2c, the expression $P<0.001$ should be $P=0.001$.
At the bottom of Figs. 2 and 3, the labels A, B, and C should be lowercase.

The labels (b) and (c) in the third sentence in the Figure 3 caption should be switched so that the sentence reads: CRP level (c) was more highly correlated with disease activity $(r=0.811)$ than was ESR (b) $(r=0.585)$.

The online version of the original article can be found under doi: 10.1007/s10620-008-0514-5.

J. J. Park · J. H. Cheon $(\bowtie) \cdot$ D. H. Kim ·

E. S. Kim · T. I. Kim - W. H. Kim

Division of Gastroenterology, Department of Internal Medicine, Institute of Gastroenterology, Yonsei University College of

Medicine, 134 Shinchon-dong, Seodaemun-gu, Seoul,

South Korea

e-mail: geniushee@yuhs.ac

B. Y. Kim · K. R. Lee

Seoul Medical Science, Seoul, South Korea 


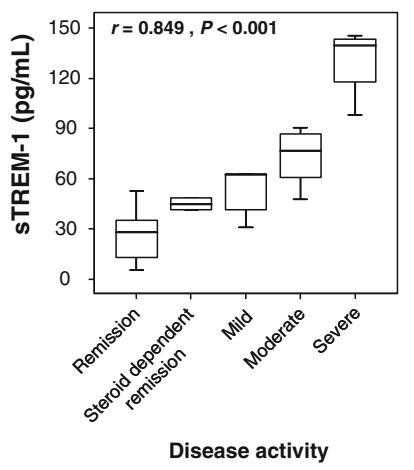

a STREM-1 versus disease activity

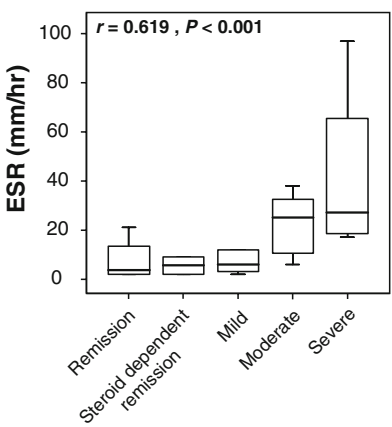

Disease activity

b ESR versus disease activity

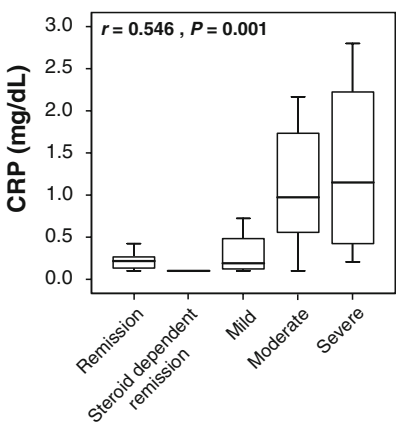

Disease activity

c CRP versus disease activity
Fig. 2 Correlation analysis of disease activity in ulcerative colitis. Soluble TREM-1 level (a) was more highly correlated with disease activity $(r=0.849)$ than was ESR $(\mathbf{b})(r=0.619)$ or CRP (c) $(r=0.546)$. The top and the bottom of each box are the 75th and 25th percentiles, respectively. The top and bottom of each bar are the 90th and 10th percentiles, respectively. Correlation between variables was assessed using Spearman's rank correlation

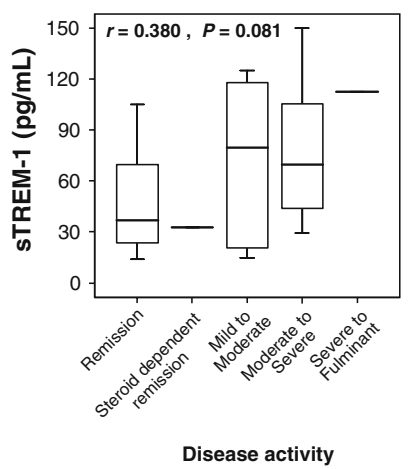

a sTREM-1 versus disease activity

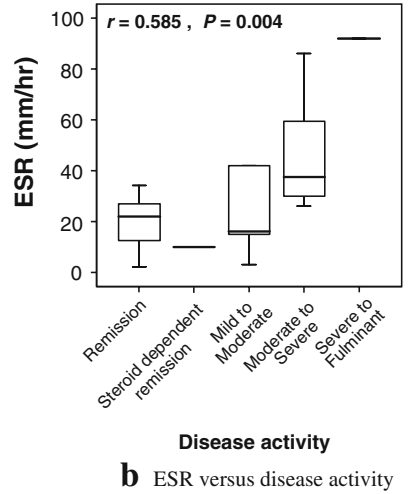

b ESR versus disease activity

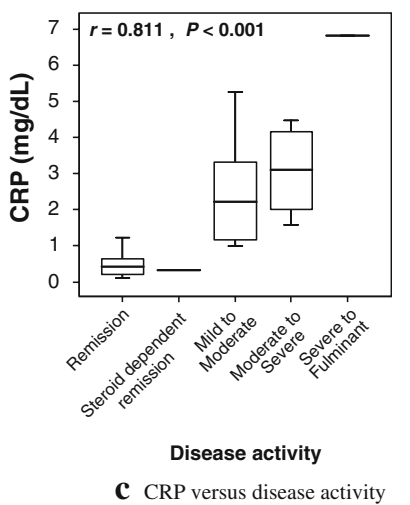

Fig. 3 Correlation analysis of disease activity in Crohn's disease. There was a trend toward lower soluble TREM-1 level (a) in those with remission compared with those without $(r=0.380)$, but this relationship did not reach statistical significance $(P=0.081)$. CRP level (c) was more highly correlated with disease activity $(r=0.811)$ than was ESR (b) $(r=0.585)$. The top and the bottom of each box are the 75 th and 25 th percentiles, respectively. The top and the bottom of each bar are the 90th and 10th percentiles, respectively. Correlation between variables was assessed using Spearman's rank correlation 